\title{
A New Proof of M. Herman's Theorem
}

\author{
K. M. Khanin and Ya. G. Sinai \\ L. D. Landau Institute for Theoretical Physics, Kosygin Str. 2, SU-117334 Moscow, \\ V-334, USSR
}

Dedicated to Walter Thirring on his $60^{\text {th }}$ birthday

\begin{abstract}
A new proof of the M. Herman theorem on the smooth conjugacy of a circle map is presented here. It is based on the thermodynamic representation of dynamical systems and the study of the ergodic properties for the corresponding radom variables.
\end{abstract}

\section{Introduction}

In this paper we present a new proof of M. Herman's famous theorem about smooth conjugacy of diffeomorphisms of the circle to rotations. Our proof is based on a version of thermodynamic formalism which was used earlier for the study of Feigenbaum's mappings of the interval (see [1]) and later for some critical mappings of the circle (see $[2,3]$ ).

Consider a strictly monotone continuous function $\varphi$ such that $\varphi(x+1)$ $=\varphi(x)+1$. It defines a homeomorphism of $S^{1}$ through the equation: $T_{\varphi} x=\{\varphi(x)\}$, $x \in[0 ; 1)$. Denote the rotation number of $T_{\varphi}$ by $\varrho$.

Assumptions. A.1. $\varphi \in C^{2+\gamma}, \gamma>0, \varphi^{\prime} \geqq$ const $>0$;

A.2. $\varrho$ is irrational and if

$$
\varrho=\left[k_{1}, k_{2}, \ldots, k_{n}, \ldots\right]
$$

is the expansion of $\varrho$ into continued fraction, then $k_{n} \leqq$ const $n^{\nu}, v>0$.

If $\varrho$ is irrational and $\varphi \in C^{2}$, then Denjoy's classical theorem states that $T_{\varphi}$ is topologically isomorphic to the rotation with angle $\varrho$. In other words there exists a strictly monotone continuous function $\psi, \psi(x+1)=\psi(x)+1$, such that $\psi(\varphi(x))$ $=\psi(x)+\varrho$. If we denote $R_{\varrho}=T_{\varphi_{0}} \varphi_{0}=x+\varrho$, then the last equality means $T_{\psi} T_{\varphi}$ $=R_{\varrho} T_{\psi}$.

Herman's Theorem. Under the Assumptions A.1, A.2 the function $\psi \in C^{1}$.

For us it will be convenient to prove a statement equivalent to Herman's theorem. This is pointed out in Arnold's paper [11]. Let us write the equation for the density $\pi(x)$ of an invariant absolutely continuous measure, provided that such 
a measure exists:

$$
\frac{\pi\left(T_{\varphi}(x)\right)}{\pi(x)}=\frac{1}{\varphi^{\prime}(x)}
$$

Herman's Theorem. Under the Assumptions A.1, A.2, Eq.(1) has a continuous strictly positive solution.

The equivalence of the two formulations follows from the equality $\psi(x)$ $=\int_{0}^{x} \pi(y) d y$. Take any point $x_{0} \in S^{1}$ and its semi-trajectory $O=\left\{x_{n}\right\}_{0}^{\infty}, x_{n}=T_{\varphi}^{n} x_{0}$ which is everywhere dense on $S^{1}$. Put $\pi\left(x_{0}\right)=1$ and $\pi\left(x_{i}\right)=\prod_{k=0}^{i-1}\left(\varphi^{\prime}\left(x_{k}\right)\right)^{-1}$. Thus, the function $\pi$ is defined on $O$ and satisfies there (1). We shall show that it is continuous on $O$ and can be continued to a positive continuous function on the whole circle.

Our method uses the ideas of the renormalization group theory in the theory of dynamical systems, as it is presented in $[4,5]$. We hope that it can also be useful for the critical mappings of the circle.

The first proof of Herman's theorem was published in [6]. Later there appeared shorter versions (see [7]). For us the exposition presented in the dissertation by Yoccoz [8] was very useful.

In contrast to the positive results of this paper, there are examples [12] of $C^{2}$ diffeomorphisms arbitrarily close to a rotation which are not $C^{1}$ conjugate to a rotation, even if their rotation numbers satisfy the hypothesis given here.

\section{The Symbolic Representations of Real Numbers Generated by $T_{\varphi}$}

Denote by $\varrho_{n}=\frac{p_{n}}{q_{n}}$ the $n^{\text {th }}$ approximant of $\varrho$, i.e. $\varrho_{n}=\left[k_{1}, k_{2}, \ldots, k_{n}\right]$. The numbers $p_{n}, q_{n}$ satisfy the recurrence relations

$$
\begin{array}{lll}
p_{n+1}=k_{n+1} p_{n}+p_{n-1}, & p_{1}=1, & p_{0}=0, \\
q_{n+1}=k_{n+1} q_{n}+q_{n-1}, & q_{1}=k_{1}, & q_{0}=1 .
\end{array}
$$

Take any integer $m \leqq q_{n+1}$. It can be written in the form

$$
m=a_{n} q_{n}+a_{n-1} q_{n-1}+\ldots+a_{0} q_{0},
$$

where $0 \leqq a_{i} \leqq k_{i+1}$. This representation is nonunique. For any $x_{0} \in S^{1}$, let us denote by $C_{0}^{(n)}\left(x_{0}\right)$ the closed intervals whose end-points are $x_{0}, x_{q_{n}}, C_{i}^{(n)}\left(x_{0}\right)=T_{\varphi}^{i} C_{0}^{(n)}\left(x_{0}\right)$. The following lemma is well-known.

Lemma 1. For every $n$ the intervals $C_{i}^{(n)}\left(x_{0}\right), 0 \leqq i<q_{n+1}, C_{j}^{(n+1)}\left(x_{0}\right), 0 \leqq j<q_{n}$ are mutually disjoint except the end-points, and cover the whole circle.

Introduce the partition $\xi_{n}$ of $S^{1}$ whose elements are $C_{i}^{(n)}\left(x_{0}\right), 0 \leqq i \leqq q_{n+1}-1$, $C_{j}^{(n+1)}\left(x_{0}\right), 0 \leqq j \leqq q_{n}-1$. The partitions $\xi_{n}$ are monotone-increasing, $\xi_{n} \leqq \xi_{n+1}$ in the sense that each $C_{\xi_{n}}$ consists of several $C_{\xi_{n+1}}$. To be more precise each $C_{j}^{(n+1)}\left(x_{0}\right)$, $0 \leqq j<q_{n}$ is also an element of the partition $\xi_{n+1}$. But each $C_{i}^{(n)}\left(x_{0}\right)$ is partitioned into $\left(k_{n+2}+1\right)$ elements of $\xi_{n+1}$ because we have the equality:

$$
C_{i}^{(n)}\left(x_{0}\right)=C_{i}^{(n+2)}\left(x_{0}\right) \cup \bigcup_{j=0}^{k_{n}+2-1} C_{i+q_{n}+j q_{n+1}}^{(n+1)}\left(x_{0}\right) .
$$


In other words if $O_{n}=\underbrace{q_{n+1}+q_{n}-1 x_{2}}_{i=0}$, then each $C_{i}^{(n)}$ contains exactly $k_{n+2}$ new points of $O_{n+1}$. It will also be important that $C_{i}^{(n)}\left(x_{0}\right)=C_{0}^{(n)}\left(x_{i}\right)$. One can construct a unique symbolic representation of any $x \in S^{1}$ not belonging to the set of end-points of all $C_{i}^{(n)}\left(x_{0}\right), 0 \leqq i<q_{n+1}$, using some inductive procedure. Put $a_{n+1}=A$ if $x \in C_{i}^{(n+1)}\left(x_{0}\right)$, $0 \leqq i<q_{n}$ and $a_{n+1}=k_{n+2}-j$ if $x \in C_{i+q_{n}+j q_{n+1}}^{(n+1)}\left(x_{0}\right) \subset C_{i}^{(n)}\left(x_{0}\right), 0 \leqq i<q_{n+1}, 0 \leqq j$ $<k_{n+2}[$ see (3)]. For

$$
x \in C_{i}^{(n)}\left(x_{0}\right)-\bigcup_{j=0}^{k_{n}+2-1} C_{i+q_{n}+j q_{n+2}}^{(n+1)}\left(x_{0}\right)=C_{i}^{(n+2)}\left(x_{0}\right) \text { put } a_{n+1}=0 .
$$

Thus, we write

$$
x \leftrightarrow\left(a_{0}, a_{1}, \ldots, a_{n}, \ldots\right)=a,
$$

where $a_{i}=A, 0,1, \ldots, k_{i+1}, i \neq 0, a_{0}=A, 0, \ldots, k_{1}-1$. The Lebesgue measure $\ell$ induces a probability measure on the space of all admissible sequences $a=\left(a_{0}, a_{1}, \ldots, a_{n}, \ldots\right)$. It is easy to see that the only restriction on the word $a$ is the following one: $a_{n+1}=A$ iff $a_{n}=0$. This is a "hard core" type condition in statistical mechanics. Remark that each element of the partition $\xi_{n}$ corresponds under this representation to a finite word $\left(a_{0}, a_{1}, \ldots, a_{n}\right)$ of length $(n+1)$. In particular, the words $(0, A, \ldots, 0, A)$ and $(A, 0, \ldots, A, 0)$ correspond to the elements $C_{0}^{(n)}, C_{0}^{(n+1)}$, respectively. The proof of Herman's theorem will be based on the ergodic properties of the sequence of random variables $a_{n}$ with respect to the measure induced by the Lebesgue measure.

\section{The Formulation of the Main Lemmas and the Derivation of Herman's Theorem}

Let us recall the

Denjoy Lemma (see [9]). There exists an absolute constant $C>0$ such that for every $x_{0} \in S^{1}, n>0$,

$$
e^{-C} \leqq \prod_{i=0}^{q_{n}-1} \varphi^{\prime}\left(x_{i}\right) \leqq e^{C}
$$

Henceforth all constants depend on $\varphi$ but do not depend on $n$. Assume that Denjoy's lemma can be strengthened in the following way:

$$
e^{-\varepsilon_{n}} \leqq \prod_{i=0}^{q_{n}-1} \varphi^{\prime}\left(x_{i}\right) \leqq e^{\varepsilon_{n}},
$$

where $\sum_{n} k_{n+1} \varepsilon_{n}<\infty$. Then it implies Herman's theorem. Indeed, let us consider $\pi\left(x_{i}\right)$ for $0 \leqq i<q_{n+1}$. Take $C_{i}^{(n)}, 0 \leqq i<q_{n+1}$, whose end-points are $x_{i}, x_{i+q_{n}}$. It contains inside itself $k_{n+2}$ points $x_{i+q_{n}+j q_{n+1}}, 1 \leqq j \leqq k_{n+2}$, and

$$
\begin{gathered}
e^{-\varepsilon_{n+1}} \leqq \frac{\pi\left(x_{i+q_{n}+j q_{n+1}}\right)}{\pi\left(x_{i+q_{n}+(j-1) q_{n+1}}\right)}=\left(\prod_{t=i+q_{n}+(j-1) q_{n+1}}^{i+q_{n}+j q_{n+1}-1} \varphi^{\prime}\left(x_{t}\right)\right)^{-1} \leqq e^{\varepsilon_{n+1}} \\
e^{-\varepsilon_{n}} \leqq \frac{\pi\left(x_{i+q_{n}}\right)}{\pi\left(x_{i}\right)} \leqq e^{\varepsilon_{n}}
\end{gathered}
$$


It shows that $\pi\left(x_{i+q_{n}+j q_{n+1}}\right)$ differs from $\pi\left(x_{i}\right)$ by a multiplicative factor which is bounded by $e^{ \pm\left(k_{n}+2 \varepsilon_{n+1}+\varepsilon_{n}\right)}$. Thus, for every $x_{j} \in C_{i}^{(n)}$, we have

$$
\begin{aligned}
& \exp \left\{-\varepsilon_{n}-\sum_{m \geqq n}\left(k_{m+2}+1\right) \varepsilon_{m+1}\right\} \leqq \frac{\pi\left(x_{j}\right)}{\pi\left(x_{i}\right)} \\
& \leqq \exp \left\{\varepsilon_{n}+\sum_{m \geqq n}\left(k_{m+2}+1\right) \varepsilon_{m+1}\right\},
\end{aligned}
$$

which implies the desired continuity and positivity of $\pi$ on $O$. Now we formulate some lemmas which will give a stronger version of Denjoy's lemma.

For any $y \in S^{1}$ consider $y \in C_{i_{0}}^{(n)}\left(x_{0}\right) \subset C_{i_{k}}^{(n-k)}\left(x_{0}\right)$.

Lemma 2. There exists a constant $\lambda<1$ not depending on $y, k, n, x_{0}$ such that

$$
\ell\left(C_{i_{0}}^{(n)}\left(x_{0}\right)\right) \leqq \lambda^{k} \ell\left(C_{i_{k}}^{(n-k)}\left(x_{0}\right)\right) .
$$

Put $H_{m}\left(y_{0}\right)=\sum_{i=0}^{q_{m}-1} \log \varphi^{\prime}\left(y_{i}\right)$, and take $y_{0}^{(1)}, y_{0}^{(2)} \in C_{i}^{(n)}\left(x_{0}\right)$.

Lemma 3. $\left|H_{n-k}\left(y_{0}^{(1)}\right)-H_{n-k}\left(y_{0}^{(2)}\right)\right| \leqq$ const $\lambda^{k}$, where the constant $\lambda$ is the same as in Lemma 2.

Put $\Delta^{(n)} x_{j}=\left|x_{j+q_{n+1}}-x_{j}\right|$, and

$$
b_{n}=\max \left\{\max _{0 \leqq p<q_{n}, x_{0}}\left|\sum_{j=0}^{p} \frac{\varphi^{\prime \prime}\left(x_{j}\right)}{\varphi^{\prime}\left(x_{j}\right)} \Delta^{(n)} x_{j}\right| ; \lambda^{n \gamma}\right\} .
$$

Lemma 4. $\left|H_{n}\left(y_{0}\right)-H_{n}\left(x_{0}\right)\right| \leqq$ const $\cdot b_{n}$ for every $y_{0} \in C_{0}^{(n+1)}\left(x_{0}\right)$.

$$
\left[\frac{n}{2}\right]
$$

Lemma 5. $\left|H_{n}\left(y_{0}\right)-H_{n}\left(x_{0}\right)\right| \leqq$ const $\sum_{k=0} b_{n-k}(n-k)^{v}+$ const $n^{v+1} \lambda^{n / 2}$ for arbitrary $x_{0}, y_{0} \in S^{1}$.

\section{Lemma 6.}

$$
\left|\sum_{j=0}^{q_{n}-1} \frac{\varphi^{\prime \prime}\left(x_{j}\right)}{\varphi^{\prime}\left(x_{j}\right)} \Delta^{(n)} x_{j}\right| \leqq \text { const } \lambda_{1}^{\sqrt{n}}, \quad \lambda<\lambda_{1}<1 .
$$

Lemma 7. For every $0 \leqq p<q_{n}$,

$$
\left|\sum_{j=0}^{p} \frac{\varphi^{\prime \prime}\left(x_{j}\right)}{\varphi^{\prime}\left(x_{j}\right)} \Delta^{(n)} x_{j}\right| \leqq \text { const } n^{2 v+2} \lambda_{2}^{V n}, \quad \lambda_{1}<\lambda_{2}<1 .
$$

Lemmas 4-7 give the stronger version of Denjoy's lemma alluded to before. We may choose $\varepsilon_{n}=$ const $n^{3 v+3} \lambda_{2}^{\sqrt{n}}$ in (4). All lemmas are proved in the next two sections.

\section{Proof of Lemmas 2-5}

Proof of Lemma 2. Take an element $C_{i}^{(n-1)}$ of the partition $\xi^{(n-1)}$. The partition $\xi^{(n)}$ decomposes $C_{i}^{(n-1)}$ into $\left(k_{n+1}+1\right)$ elements. One of them is $C_{i}^{(n+1)}$, while the others belong to the trajectory of the $C_{0}^{(n)}$. Take first $C_{i}^{(n+1)}$. There exists some 
$C_{j}^{(n)} C C_{i}^{(n-1)}$ such that $C_{j+q_{n}}^{(n)} \supset C_{i}^{(n+1)}$. By Denjoy's lemma

$$
e^{-C} \leqq \frac{\ell\left(C_{j+q_{n}}^{(n)}\right)}{\ell\left(C_{j}^{(n)}\right)} \leqq e^{C}
$$

and

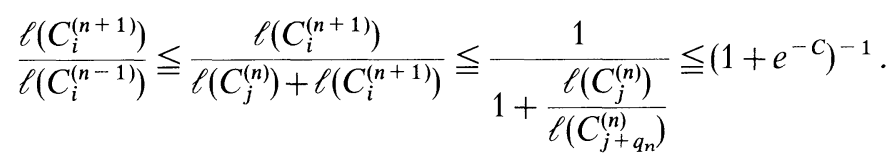

Assume now that $k_{n+1}>1$ and take some $C_{j}^{(n)} C C_{i}^{(n-1)}$. Then $C_{j \pm q_{n}}^{(n)} C C_{i}^{(n-1)}$ for at least one choice of sign. Then again with the help of Denjoy's lemma

$$
\frac{\ell\left(C_{j}^{(n)}\right)}{\ell\left(C_{i}^{(n-1)}\right)} \leqq \frac{\ell\left(C_{j}^{(n)}\right)}{\ell\left(C_{j}^{(n)}\right)+\ell\left(C_{j \pm q_{n}}^{(n)}\right)} \leqq\left(1+e^{-C}\right)^{-1}
$$

Now we discuss the case $k_{n+1}=1$. Here $C_{i}^{(n-1)}=C_{i}^{(n+1)}+C_{i+q_{n-1}}^{(n)}$ and the ratio $\frac{\ell\left(C_{i+q_{n-1}}^{(n)}\right)}{\ell\left(C_{i}^{(n-1)}\right)}$ can be very close to 1 . The partition $\xi^{(n+1)}$ decomposes $C_{i+q_{n-1}}^{(n)}$ into $\left(k_{n+2}+1\right)$ elements. As above the ratio of the lengths of these elements to $\ell\left(C_{i}^{(n-1)}\right)$ is less than $\left(1+e^{-C}\right)^{-1}$ if $k_{n+2}>1$. If $k_{n+2}=1$, then we have $C_{i+q_{n-1}+q_{n}}^{(n+1)} C C_{i+q_{n-1}}^{(n)}$ $C C_{i}^{(n-1)}$ and

$$
\frac{\ell\left(C_{i+q_{n-1}+q_{n}}^{(n+1)}\right)}{\ell\left(C_{i}^{(n-1)}\right)} \leqq \frac{\ell\left(C_{\left.i+q_{n-1}+q_{n}\right)}^{(n+1)}\right)}{\ell\left(C_{i}^{(n+1)}\right)+\ell\left(C_{i+q_{n-1}+q_{n}}^{(n+1)}\right.} \leqq\left(1+e^{-2 C}\right)^{-1} .
$$

Thus, after two steps the ratio in all cases becomes less than $\left(1+e^{-2 C}\right)^{-1}$. This immediately gives the statement of the lemma.

Proof of Lemma 3. Take $y_{0}^{(1)}, y_{0}^{(2)} \subset C_{i}^{(n)}\left(x_{0}\right) \subset C_{j}^{(n-k)}\left(x_{0}\right)$ or $C_{j}^{(n-k+1)}\left(x_{0}\right)$. First we remark that $C_{i}^{(n)}\left(x_{0}\right)=C_{0}^{(n)}\left(x_{i}\right)$. Thus, we may replace $x_{0}$ by $x_{i}$ and assume that $y_{0}^{(1)}, y_{0}^{(2)} \in C_{0}^{(n)}(x) \subset C_{0}^{(n-m)}(x)$, where $m=k$ or $k-1$ is an even number. Then $y_{i}^{(1)}, y_{i}^{(2)} \in C_{i}^{(n)}(x) \subset C_{i}^{(n-m)}(x)$. Now we can write using Lemma 2 ,

$$
\begin{gathered}
\left|H_{n-k}\left(y_{0}^{(1)}\right)-H_{n-k}\left(y_{0}^{(2)}\right)\right| \leqq \sum_{i=0}^{q_{n-k}-1}\left|\log \varphi^{\prime}\left(y_{i}^{(1)}\right)-\log \varphi^{\prime}\left(y_{i}^{(2)}\right)\right| \\
\leqq \max _{x \in S^{1}}\left|\frac{\varphi^{\prime \prime}(x)}{\varphi^{\prime}(x)}\right| \cdot \max _{i} \frac{\ell\left(C_{i}^{(n)}(x)\right)}{\ell\left(C_{i}^{(n-m)}(x)\right)} \leqq \mathrm{const} \lambda^{m} . \quad \text { QED} .
\end{gathered}
$$

Proof of Lemma 4. Let $I=\sum_{i=0}^{q_{n}-1}\left[\log \varphi^{\prime}\left(y_{i}\right)-\log \varphi^{\prime}\left(x_{i}\right)\right]$. We have

$$
I=\sum_{i=0}^{q_{n}-1} \frac{\varphi^{\prime \prime}\left(x_{i}\right)}{\varphi^{\prime}\left(x_{i}\right)}\left(y_{i}-x_{i}\right)+\sum_{i=0}^{q_{n}-1} \alpha_{i}\left(y_{i}-x_{i}\right)^{1+\gamma}=I_{1}+I_{2},\left|\alpha_{i}\right| \leqq \text { const } .
$$

For $I_{2}$ we have a trivial estimate with the help of Lemma 2 for $k=n$ :

$$
\left|I_{2}\right| \leqq \text { const } \max \left|y_{i}-x_{i}\right|^{\gamma} \leqq \text { const } \lambda^{n \gamma} \text {. }
$$


Introduce $\varrho_{i}=\frac{y_{i}-x_{i}}{\Delta^{(n)} x_{i}}$.

$$
\begin{aligned}
\varrho_{i+1} & =\frac{\varphi^{\prime}\left(x_{i}\right)\left(y_{i}-x_{i}\right)+\frac{1}{2} \varphi^{\prime \prime}\left(x_{i}\right)\left(y_{i}-x_{i}\right)^{2}+\alpha_{i}^{\mathrm{I}}\left(y_{i}-x_{i}\right)^{2+\gamma}}{\varphi^{\prime}\left(x_{i}\right) \Delta^{(n)} x_{i}+\frac{1}{2} \varphi^{\prime \prime}\left(x_{i}\right)\left(\Delta^{(n)} x_{i}\right)^{2}+\alpha_{i}^{\mathrm{II}}\left(\Delta^{(n)} x_{i}\right)^{2+\gamma}} \\
& =\varrho_{i} \frac{1+\frac{1}{2} \frac{\varphi^{\prime \prime}\left(x_{i}\right)}{\varphi^{\prime}\left(x_{i}\right)}\left(y_{i}-x_{i}\right)+\alpha_{i}^{\mathrm{III}}\left(y_{i}-x_{i}\right)^{1+\gamma}}{1+\frac{1}{2} \frac{\varphi^{\prime \prime}\left(x_{i}\right)}{\varphi^{\prime}\left(x_{i}\right)} \Delta^{(n)} x_{i}+\alpha_{i}^{(\mathrm{IV})}\left(\Delta^{(n)} x_{i}\right)^{1+\gamma}} .
\end{aligned}
$$

In the above (and henceforth) $\alpha_{i}^{\mathrm{I}}, \alpha_{i}^{\mathrm{II}}, \ldots$ are some uniformly bounded numbers. Put

$$
\varrho_{i}^{\prime}=\varrho_{i} \prod_{j<i}\left[1+\frac{1}{2} \frac{\varphi^{\prime \prime}\left(x_{j}\right)}{\varphi^{\prime}\left(x_{j}\right)} \Delta^{(n)} x_{j}+\alpha_{i}^{(\mathrm{IV})}\left(\Delta^{(n)} x_{j}\right)^{1+\gamma}\right]=\varrho_{i}\left(1+b_{i}^{\prime}\right) .
$$

For $b_{i}^{\prime}$ we have the estimate $\left|b_{i}^{\prime}\right| \leqq$ const $b_{n}$. Furthermore,

$$
\varrho_{i+1}^{\prime}=\varrho_{i}^{\prime}\left[1+\frac{1}{2} \frac{\varphi^{\prime \prime}\left(x_{i}\right)}{\varphi^{\prime}\left(x_{i}\right)} \varrho_{i}^{\prime}\left(1+b_{i}^{\prime}\right)^{-1} \Delta^{(n)} x_{i}+\alpha_{i}^{\mathrm{III}}\left(\varrho_{i}^{\prime}\left(1+b_{i}^{\prime}\right)^{-1}\right)^{1+\gamma}\left(\Delta^{(n)} x_{i}\right)^{1+\gamma}\right]
$$

and

$$
\begin{aligned}
& \frac{1}{\varrho_{i+1}^{\prime}}=\frac{1}{\varrho_{i}^{\prime}}\left[1-\frac{1}{2} \frac{\varphi^{\prime \prime}\left(x_{i}\right)}{\varphi^{\prime}\left(x_{i}\right)} \varrho_{i}^{\prime}\left(1+b_{i}^{\prime}\right)^{-1} \Delta^{(n)} x_{i}+\alpha_{i}^{(\mathrm{V})}\left(\varrho_{i}^{\prime}\left(1+b_{i}^{\prime}\right)^{-1}\right)^{1+\gamma}\left(\Delta^{(n)} x_{i}\right)^{1+\gamma}\right], \\
& \frac{1}{\varrho_{i+1}^{\prime}}=\frac{1}{\varrho_{i}^{\prime}}-\frac{1}{2} \frac{\varphi^{\prime \prime}\left(x_{i}\right)}{\varphi^{\prime}\left(x_{i}\right)}\left(1+b_{i}^{\prime}\right)^{-1} \Delta^{(n)} x_{i}+\alpha_{i}^{(\mathrm{V})}\left(\varrho_{i}^{\prime}\right)^{\gamma}\left(1+b_{i}^{\prime}\right)^{-1-\gamma}\left(\Delta^{(n)} x_{i}\right)^{1+\gamma} .
\end{aligned}
$$

This yields

$$
\left|\frac{1}{\varrho_{i}^{\prime}}-\frac{1}{\varrho_{0}^{\prime}}\right| \leqq \text { const }\left|\sum_{j<i} \frac{\varphi^{\prime \prime}\left(x_{j}\right)}{\varphi^{\prime}\left(x_{j}\right)} \Delta^{(n)} x_{j}\right|+\text { const } b_{n} \leqq \text { const } b_{n},
$$

and

$$
\begin{aligned}
& \left|\varrho_{0}^{\prime}-\varrho_{i}^{\prime}\right| \leqq \varrho_{0}^{\prime} \varrho_{i}^{\prime} \text { const } b_{n} \leqq \text { const } b_{n} \varrho_{0}, \\
& \left|\varrho_{0}-\varrho_{i}\right| \leqq \text { const } b_{n} \varrho_{0} .
\end{aligned}
$$

The last inequality shows that $\varrho_{i}$ differs from $\varrho_{0}$ by a very small number (assuming that $b_{n}$ are small enough), i.e. the mapping $T_{\varphi}^{i}: C_{0}^{(n+1)} \rightarrow C_{i}^{(n+1)}$ can be well enough approximated by a linear map. Now we can write

$$
\begin{aligned}
\left|I_{1}\right| & =\left|\sum_{i=0}^{q_{n}-1} \frac{\varphi^{\prime \prime}\left(x_{i}\right)}{\varphi^{\prime}\left(x_{i}\right)}\left(y_{i}-x_{i}\right)\right|=\left|\sum_{i=0}^{q_{n}-1} \frac{\varphi^{\prime \prime}\left(x_{i}\right)}{\varphi^{\prime}\left(x_{i}\right)} \Delta^{(n)} x_{i} \varrho_{i}\right| \\
& \leqq \varrho_{0}\left|\sum_{i=0}^{q_{n}-1} \frac{\varphi^{\prime \prime}\left(x_{i}\right)}{\varphi^{\prime}\left(x_{i}\right)} \Delta^{(n)} x_{i}\right|+\left|\sum_{i=0}^{q_{n}-1} \frac{\varphi^{\prime \prime}\left(x_{i}\right)}{\varphi^{\prime}\left(x_{i}\right)} \Delta^{(n)} x_{i}\left(\varrho_{i}-\varrho_{0}\right)\right| \\
& \leqq \text { const } b_{n} \varrho_{0} . \quad \text { QED. }
\end{aligned}
$$


Proof of Lemma 5. Take $x_{0}$ and construct the sequence of the intervals $C_{i}^{(m)}\left(x_{0}\right)$, $m=n, n+1$. Then $y_{0} \in C_{i}^{(n+1)}\left(x_{0}\right)$ for some $i, 0 \leqq i<q_{n}$, or $y_{0} \in C_{j}^{(n)}\left(x_{0}\right)$ for some $j, 0 \leqq j$ $<q_{n+1}$. In the first case

$$
\begin{aligned}
J_{n}= & H_{n}\left(y_{0}\right)-H_{n}\left(x_{0}\right)=\sum_{s=0}^{q_{n}-1-i}\left[\log \varphi^{\prime}\left(y_{s}\right)-\log \varphi^{\prime}\left(x_{s+i}\right)\right] \\
& +\sum_{s=q_{n}-i}^{q_{n}-1}\left[\log \varphi^{\prime}\left(y_{s}\right)-\log \varphi^{\prime}\left(x_{s-q_{n}+i}\right)\right] .
\end{aligned}
$$

Remark that $y_{q_{n}-i} \in C_{q_{n}}^{(n+1)}\left(x_{0}\right) \subset C_{0}^{(n)}\left(x_{0}\right)$ and recall (see Sect. 2) that $C_{j}^{(m)}\left(x_{i}\right)$ $=C_{i+j}^{(m)}\left(x_{0}\right)$. Therefore, each of the sums has the form $J_{r}^{\prime}=\sum_{s=0}^{r}\left[\log \varphi^{\prime}\left(z_{s}^{(1)}\right)\right.$ $\left.-\log \varphi^{\prime}\left(z_{s}^{(2)}\right)\right]$, where $z_{0}^{(1)}, z_{0}^{(2)} \in C_{0}^{(n)}(x)$ for some $x$ and $0 \leqq r \leqq q_{n}-1$. Write (see Sect. 2)

$$
r=a_{n-1} q_{n-1}+a_{n-2} q_{n-2}+\ldots+a_{0} q_{0},
$$

where $0 \leqq a_{i} \leqq k_{i+1}$. Then

$$
J_{r}^{\prime}=\sum_{j: a_{J} \neq 0} \sum_{i=1}^{a_{j}} \sum_{\substack{a_{n-1} q_{n-1}+\ldots+a_{j+1} q_{j+1}+(i-1) q_{j} \leqq s \\<a_{n-1} q_{n-1}+\ldots+a_{j}+1 q_{j+1}+i q_{j}}}\left[\log \varphi^{\prime}\left(z_{s}^{(1)}\right)-\log \varphi^{\prime}\left(z_{s}^{(2)}\right)\right] .
$$

Consider each inner sum separately. For every $s$ the points $z_{s}^{(1)}, z_{s}^{(2)}$ belong to some $C_{0}^{(n)}$. Using Lemma 4 for long cycles $\left(j \geqq \frac{n}{2}\right)$ and Lemma 3 for short cycles $\left(j<\frac{n}{2}\right)$
we can write:

\section{$\left[\frac{n}{2}\right]$}

$$
\begin{aligned}
\left|J_{r}^{\prime}\right|= & \left|\sum_{j \geq \frac{n}{2}}\right|+\left|\sum_{j<\frac{n}{2}}\right| \leqq \operatorname{const} \sum_{k=0} b_{n-k}(n-k)^{v}+ \\
& \text { const } n^{v+1} \lambda^{n / 2},
\end{aligned}
$$

which implies the desired result.

In the second case the arguments are the same if $j<q_{n}$. If $q_{n} \ell \leqq j<q_{n}(\ell+1), 0<\ell$ $\leqq k_{n+1}$, we apply the same arguments for the differences,

$$
\sum_{s=0}^{q_{n}-1}\left[\log \varphi^{\prime}\left(y_{s-t q_{n}}\right)-\log \varphi^{\prime}\left(y_{s-(t-1) q_{n}}\right)\right], \quad 1 \leqq t \leqq \ell,
$$

and then consider the difference

$$
\sum_{s=0}^{q_{n}-1}\left[\log \varphi^{\prime}\left(x_{s}\right)-\log \varphi^{\prime}\left(y_{s-\ell q_{n}}\right)\right] .
$$

Our previous arguments also work now, and thus, we get the needed estimation. QED.

Corollary. $\left|H_{n}\left(x_{0}\right)\right|=\left|\sum_{i=0}^{q_{n}-1} \log \varphi^{\prime}\left(x_{i}\right)\right| \leqq$ const $n^{3 v+3} \lambda 2_{2}^{\sqrt{n}}$. because

Indeed, apply Lemmas 5-7 for $x_{0}$ and $y_{0}$, where $H_{n}\left(y_{0}\right)=0$. Such $y_{0}$ exists,

$$
\underbrace{\varphi(\varphi(\ldots \varphi}_{q_{n} \text { times }}(x+1) \ldots)-\underbrace{\varphi(\varphi(\ldots \varphi}_{q_{n} \text { times }}(x) \ldots)=1,
$$


and in view of the mean value theorem there exists $y_{0}$ for which

$$
\frac{d}{d x} \underbrace{\varphi(\varphi \ldots \varphi}_{q_{n} \text { times }}\left(y_{0}\right) \ldots)=\prod_{i=0}^{q_{n}-1} \varphi^{\prime}\left(y_{i}\right)=1, \quad H_{n}\left(y_{0}\right)=0 .
$$

We have used the results of Lemmas 6 and 7. Their proofs do not depend on Lemmas 4 and 5.

\section{Proofs of Lemmas 6 and 7}

Proof of Lemma 6. We have to estimate the sum

$$
I_{n}=\sum_{j=0}^{q_{n}-1} \frac{\varphi^{\prime \prime}\left(x_{j}\right)}{\varphi^{\prime}\left(x_{j}\right)} \Delta^{(n)} x_{j}
$$

Putting $k=\left[\frac{n}{2}\right]$, rewrite it in the following way:

$$
\begin{aligned}
I_{n}= & \sum_{s=0}^{q_{n-k+1}-1} \sum_{j: C_{j}^{(n+1)} \subset C_{s}^{(n-k)}} \frac{\varphi^{\prime \prime}\left(x_{j}\right)}{\varphi^{\prime}\left(x_{j}\right)} \Delta^{(n)} x_{j} \\
& +\sum_{t=0}^{q_{n-k}-1} \sum_{j: C_{j}^{(n+1)} \subset C_{t}^{(n-k+1)}} \frac{\varphi^{\prime \prime}\left(x_{j}\right)}{\varphi^{\prime}\left(x_{j}\right)} \Delta^{(n)} x_{j} .
\end{aligned}
$$

Choose an arbitrary point $y_{s}^{(n-k)}, y_{t}^{(n-k+1)}$ in each $C_{s}^{(n-k)}, C_{t}^{(n-k+1)}$. Then

$$
\begin{aligned}
I_{n}= & \sum_{s=0}^{q_{n-k+1}-1} \frac{\varphi^{\prime \prime}\left(y_{s}^{(n-k)}\right)}{\varphi^{\prime}\left(y_{s}^{(n-k)}\right)} \ell\left(C_{s}^{(n-k)}\right) p_{s}^{(n-k)} \\
& +\sum_{t=0}^{q_{n-k}-1} \frac{\varphi^{\prime \prime}\left(y_{t}^{(n-k+1)}\right)}{\varphi^{\prime}\left(y_{t}^{(n-k+1)}\right)} \ell\left(C_{t}^{(n-k+1)}\right) p_{t}^{(n-k+1)} \\
& +\sum_{s=0}^{q_{n-k+1}-1} \sum_{j}\left(\frac{\varphi^{\prime \prime}\left(x_{j}\right)}{\varphi^{\prime}\left(x_{j}\right)}-\frac{\varphi^{\prime \prime}\left(y_{s}^{(n-k)}\right)}{\varphi^{\prime}\left(y_{s}^{(n-k)}\right)}\right) \Delta^{(n)} x_{j} \\
& +\sum_{t=0}^{q_{n-k}-1} \sum_{j}\left(\frac{\varphi^{\prime \prime}\left(x_{j}\right)}{\varphi^{\prime}\left(x_{j}\right)}-\frac{\varphi^{\prime \prime}\left(y_{t}^{(n-k+1)}\right)}{\varphi^{\prime}\left(y_{t}^{(n-k+1)}\right)}\right) \Delta^{(n)} x_{j},
\end{aligned}
$$

where

$$
\begin{aligned}
p_{s}^{(n-k)} & =\frac{1}{\ell\left(C_{s}^{(n-k)}\right)} \sum_{j: C_{j}^{(n+1)} \subset C_{s}^{(n-k)}} \ell\left(C_{j}^{(n+1)}\right), \\
p_{t}^{(n-k+1)} & =\frac{1}{\ell\left(C_{t}^{(n-k+1)}\right)} \sum_{j: C_{j}^{(n+1)} \subset C_{t}^{(n-k+1)}} \ell\left(C_{j}^{(n+1)}\right) .
\end{aligned}
$$

Due to the smooth properties of $\varphi$ each of the two last sums is not more than const $\lambda^{\frac{n \gamma}{2}}$.

Assume that we have succeeded in proving that for some constant $p$ the differences

$$
\left|p_{s}^{(n-k)}-p\right|,\left|p_{t}^{(n-k+1)}-p\right| \leqq \text { const } \lambda_{1}^{\sqrt{n}}
$$


Then

$$
\begin{aligned}
& \sum_{s=0}^{q_{n-k+1}-1} \frac{\varphi^{\prime \prime}\left(y_{s}^{(n-k)}\right)}{\varphi^{\prime}\left(y_{s}^{(n-k)}\right)} \ell\left(C_{s}^{(n-k)}\right) p_{s}^{(n-k)}+\sum_{t=0}^{q_{n-k}-1} \frac{\varphi^{\prime \prime}\left(y_{t}^{(n-k+1)}\right)}{\varphi^{\prime}\left(y_{t}^{(n-k+1)}\right)} \\
& \cdot p_{t}^{(n-k+1)} \ell\left(C_{t}^{(n-k+1)}\right)=p\left[\sum_{s=0}^{q_{n-k+1}-1} \frac{\varphi^{\prime \prime}\left(y_{s}^{(n-k)}\right)}{\varphi^{\prime}\left(y_{s}^{(n-k)}\right)} \ell\left(C_{s}^{(n-k)}\right)\right. \\
& \left.+\sum_{t=0}^{q_{n-k}-1} \frac{\varphi^{\prime \prime}\left(y_{t}^{(n-k+1)}\right)}{\varphi^{\prime}\left(y_{t}^{(n-k+1)}\right)} \ell\left(C_{t}^{(n-k+1)}\right)\right]+\sum_{s=0}^{q_{n-k+1}-1} \frac{\varphi^{\prime \prime}\left(y_{s}^{(n-k)}\right)}{\varphi^{\prime}\left(y_{s}^{(n-k)}\right)} \\
& \cdot \ell\left(C_{s}^{(n-k)}\right)\left(p_{s}^{(n-k)}-p\right)+\sum_{t=0}^{q_{n-k}-1} \frac{\varphi^{\prime \prime}\left(y_{t}^{(n-k+1)}\right)}{\varphi^{\prime}\left(y_{t}^{(n-k+1)}\right)} \ell\left(C_{t}^{(n-k+1)}\right)\left(p_{t}^{(n-k+1)}-p\right) .
\end{aligned}
$$

The absolute value of the last two sums is not more than const $\lambda_{1}^{\sqrt{n}}$. The expression in square brackets is an approximation of the Riemann integral $\int \frac{\varphi^{\prime \prime}(y)}{\varphi^{\prime}(y)} d y=0$. Due to the smoothness properties of the function $\varphi$ it differs from the integral by a number with absolute value less than const $\lambda^{\frac{n \gamma}{2}}$. Thus, we have to show only inequalities (6). It will be proved via some ergodic theorem type arguments for the Markov chains.

Let us return to the increasing sequence of partitions $\xi_{n}$ and the corresponding symbolic representation which were introduced in Sect. 2. Consider the conditional probabilities $\ell\left(a_{n} \mid a_{n-1}, \ldots, a_{0}\right)=\frac{\ell\left(a_{0}, a_{1}, \ldots, a_{n}\right)}{\ell\left(a_{0}, a_{1}, \ldots, a_{n-1}\right)}$, wehere $\ell\left(a_{0}, \ldots, a_{m}\right)$ means the Lebesgue measure of the element of $\xi_{m}$ corresponding to the admissible word $\left(a_{0}, \ldots, a_{m}\right)$.

Lemma 8. The following inequality holds:

$$
e^{-\mathrm{const} \lambda^{s}} \leqq \frac{\ell\left(a_{n} \mid a_{n-1}, \ldots, a_{n-s}, a_{n-s-1}^{\prime \prime}, \ldots, a_{0}^{\prime \prime}\right)}{\ell\left(a_{n} \mid a_{n-1}, \ldots, a_{n-s}, a_{n-s-1}^{\prime}, \ldots, a_{0}^{\prime}\right)} \leqq e^{\text {const } \lambda^{s}},
$$

provided both words are admissible.

Proof. The words $\left(a_{0}^{\prime}, \ldots, a_{n-s-1}^{\prime}, a_{n-s}, \ldots, a_{n}\right),\left(a_{0}^{\prime}, \ldots, a_{n-s-1}^{\prime}, a_{n-s}, \ldots, a_{n-1}\right)$, $\left(a_{0}^{\prime}, \ldots, a_{n-s-1}^{\prime}\right)$ correspond to the intervals $C_{i_{0}}^{\left(m_{0}\right)} \subset C_{i_{1}}^{\left(m_{1}\right)} \subset C_{i_{2}}^{\left(m_{2}\right)}$, where $m_{0}=n, n+1$, $m_{1}=n-1, n, m_{2}=n-s-1, n-s$. Then the words $\left(a_{0}^{\prime \prime}, \ldots, a_{n-s-1}^{\prime \prime}, a_{n-s}, \ldots, a_{n}\right)$, $\left(a_{0}^{\prime \prime}, \ldots, a_{n-s-1}^{\prime \prime}, a_{n-s}, \ldots, a_{n-1}\right),\left(a_{0}^{\prime \prime}, \ldots, a_{n-s-1}^{\prime \prime}\right)$ correspond to the intervals $C_{i_{0}+j}^{\left(m_{0}\right)} \subset C_{i_{1}+j}^{\left(m_{1}\right)} \subset C_{i_{2}+j}^{\left(m_{2}\right)}$, where $i_{2}+j<q_{n-s-1}$, if $m_{2}=n-s$ and $i_{2}+j<q_{n-s}$ if $m_{2}$ $=n-s-1$. Denote

$$
\varrho_{k}=\frac{\ell\left(C_{i_{0}+k}^{\left(m_{0}\right)}\right)}{\ell\left(C_{i_{1}+k}^{\left(m_{1}\right)}\right)}: \frac{\ell\left(C_{i_{0}}^{\left(m_{0}\right)}\right)}{\ell\left(C_{i_{1}}^{\left(m_{1}\right)}\right)}, \quad 1 \leqq k \leqq j
$$

We have

$$
\varrho_{k+1}=\frac{\int_{C_{t_{0}+k}^{\left(m_{0}\right)}}^{\left(C_{t_{1}+k}^{\left(m_{1}\right)}\right.} \varphi^{\prime}(y) d y}{\varphi^{\prime}(y) d y}: \frac{\ell\left(C_{i_{0}}^{\left(m_{0}\right)}\right)}{\ell\left(C_{i_{1}}^{\left(m_{1}\right)}\right)} .
$$


Using the mean value theorem we can write

$$
\varrho_{k+1}=\frac{\varphi^{\prime}\left(y_{i_{0}+k}^{\left(m_{0}\right)}\right)}{\varphi^{\prime}\left(y_{i_{1}+k}^{\left(m_{1}\right)}\right)} \varrho_{k},
$$

where $y_{i}^{(m)}$ are some points of the corresponding intervals. Furthermore,

$$
\exp \left\{-\operatorname{const} \ell\left(C_{i_{1}+k}^{\left(m_{1}\right)}\right)\right\} \leqq \frac{\varphi^{\prime}\left(y_{i_{0}+k}^{\left(m_{0}\right)}\right)}{\varphi^{\prime}\left(y_{i_{1}+k}^{\left(m_{1}\right)}\right)} \leqq \exp \left\{\operatorname{const} \ell\left(C_{i_{1}+k}^{\left(m_{1}\right)}\right)\right\}
$$

Then

and

$$
\frac{\ell\left(C_{i_{0}+j}^{\left(m_{0}\right)}\right)}{\ell\left(C_{i_{1}+j}^{\left(m_{1}\right)}\right)}=\varrho_{j} \frac{\ell\left(C_{i_{0}}^{\left(m_{0}\right)}\right)}{\ell\left(C_{i_{1}}^{\left(m_{1}\right)}\right)}
$$

$$
\exp \left\{-\operatorname{const} \sum_{t=0}^{j-1} \ell\left(C_{i_{1}+t}^{\left(m_{1}\right)}\right)\right\} \leqq \varrho_{j} \leqq \exp \left\{\operatorname{const} \sum_{t=0}^{j-1} \ell\left(C_{i_{1}+t}^{\left(m_{1}\right)}\right)\right\}
$$

However, due to Lemma 2 ,

$$
\sum_{t=0}^{j-1} \ell\left(C_{i_{1}+t}^{\left(m_{1}\right)}\right) \leqq \sum_{t=0}^{j-1} \ell\left(C_{i_{2}+t}^{\left(m_{2}\right)}\right) \frac{\ell\left(C_{i_{1}+t}^{\left(m_{1}\right)}\right)}{\ell\left(C_{i_{2}+t}^{\left(m_{2}\right)}\right)} \leqq \text { const } \lambda^{s} . \quad \text { QED. }
$$

Lemma 8 shows that the sequence of random variables $\left\{a_{n}\right\}$ can be well enough approximated by the Markov chain. Consider now the conditional probabilities $\ell\left(a_{n+m}, \ldots, a_{n} \mid a_{n-1}^{\prime}, \ldots, a_{0}^{\prime}\right), \ell\left(a_{n+m}, \ldots, a_{n} \mid a_{n-1}^{\prime \prime}, \ldots, a_{0}^{\prime \prime}\right)$, provided both words

$$
\left(a_{0}^{\prime}, \ldots, a_{n-1}^{\prime}, a_{n}, \ldots, a_{n+m}\right),\left(a_{0}^{\prime \prime}, \ldots, a_{n-1}^{\prime \prime}, a_{n}, \ldots, a_{n+m}\right)
$$

are admissible.

Lemma 9. There exists an absolute constant $C_{1}>0$ such that for all $n, m$,

$$
e^{-c_{1}} \leqq \frac{\ell\left(a_{n+m}, \ldots, a_{n} \mid a_{n-1}^{\prime}, \ldots, a_{0}^{\prime}\right)}{\ell\left(a_{n+m}, \ldots, a_{n} \mid a_{n-1}^{\prime \prime}, \ldots, a_{0}^{\prime \prime}\right)} \leqq e^{c_{1}} .
$$

The proof follows easily from Lemma 8 and the equations

$$
\begin{aligned}
& \ell\left(a_{n+m}, \ldots, a_{n} \mid a_{n-1}^{\prime}, \ldots, a_{0}^{\prime}\right)=\prod_{i=0}^{m} \ell\left(a_{n+i} \mid a_{n+i-1}, \ldots, a_{n-1}^{\prime}, \ldots, a_{0}^{\prime}\right), \\
& \ell\left(a_{n+m}, \ldots, a_{n} \mid a_{n-1}^{\prime \prime}, \ldots, a_{0}^{\prime \prime}\right)=\prod_{i=0}^{m} \ell\left(a_{n+i} \mid a_{n+i-1}, \ldots, a_{n-1}^{\prime \prime}, \ldots, a_{0}^{\prime \prime}\right) .
\end{aligned}
$$

The proof of the following lemma is also simple.

Lemma 10. There exists an absolute constant $C_{2}>0$ such that for all $n, m$ and all the admissible words $\left(a_{0}^{\prime}, \ldots, a_{n-3}^{\prime}\right),\left(a_{0}^{\prime \prime}, \ldots, a_{n-3}^{\prime \prime}\right),\left(a_{n}, \ldots, a_{n+m}\right)$ :

$$
e^{-C_{2}} \leqq \frac{\ell\left(a_{n+m}, \ldots, a_{n} \mid a_{n-3}^{\prime}, \ldots, a_{0}^{\prime}\right)}{\ell\left(a_{n+m}, \ldots, a_{n} \mid a_{n-3}^{\prime \prime}, \ldots, a_{0}^{\prime \prime}\right)} \leqq e^{C_{2}} .
$$

Return now to $p_{s}^{(n-k)}, p_{t}^{(n-k+1)}$. In terms of symbolic representation they can be written in the form:

$$
p_{s}^{(n-k)}, p_{t}^{(n-k+1)}=\ell\left(a_{n}=0 \mid a_{n-k}, \ldots, a_{0}\right) .
$$


We have to estimate the difference

$$
\left|\ell\left(a_{n}=0 \mid a_{n-k}, \ldots, a_{0}\right)-\ell\left(a_{n}=0\right)\right| .
$$

Lemma 11. $\left|\ell\left(a_{n}=0 \mid a_{n-k}, \ldots, a_{0}\right)-\ell\left(a_{n}=0\right)\right| \leqq$ const $\lambda_{3}^{\sqrt{k}}$ for some constant $\lambda_{3}<1$.

Lemma 11 is a Markov ergodic theorem and it can be proved by the methods of the Markov chain theory. This technique is well-known (see [10]); thus, we shall describe only the main steps.

Fix an integer $m, m \sim \sqrt{k}$ and introduce a new probability measure on the words

$$
\begin{aligned}
\tilde{a}= & \left(a_{n}, a_{n-1}, \ldots, a_{n-m+3}, a_{n-m}, \ldots, a_{n-2 m+3},\right. \\
& \left.a_{n-2 m}, \ldots, a_{n-3 m+3}, \ldots, a_{n-(i-1) m}, \ldots, a_{n-i m+3}, a_{n-i m}, \ldots, a_{0}\right)
\end{aligned}
$$

by the formula

$$
\begin{array}{r}
\ell^{\prime}(\tilde{a})=\ell\left(a_{0}, \ldots, a_{n-i m}\right) \ell\left(a_{n-(i-1) m}, \ldots, a_{n-i m+3} \mid a_{n-i m}, \ldots, a_{0}\right) \\
\quad \cdot \prod_{j=0}^{i-2} \ell\left(a_{n-j m}, \ldots, a_{n-(j+1) m+3} \mid a_{n-(j+1) m}, \ldots, a_{n-(j+2) m+3}\right) .
\end{array}
$$

Here $i \sim \sqrt{k}$. This measure is the one of the Markov chain with memory $m$. It follows easily from Lemma 8 that

$$
\exp \left(-\operatorname{const} \lambda^{m} \cdot i\right) \leqq \frac{\ell^{\prime}(\tilde{a})}{\ell(\tilde{a})} \leqq \exp \left(\text { const } \lambda^{m} \cdot i\right)
$$

If we consider the Markov transition operator corresponding to $\ell^{\prime}$ for the transition to $m$ steps, then it follows from Lemma 10 that it is a contraction with a coefficient uniformly less than 1 . Then the usual ergodic theorem for Markov chains shows that the difference between the conditional probabilities $\ell\left(a_{n} \mid a_{n-i m}, \ldots, a_{0}\right)$ for different $a_{n-i m}, \ldots, a_{0}$ is less than $\lambda_{4}^{i}, \lambda_{4}<1$. This gives the desired result. QED.

Proof of Lemma 7. Lemma 7 is derived from Lemma 6 in a similar way as Lemma 5 is derived from Lemma 4. Consider the sums

$$
I_{n-2 i}=\sum_{j=0}^{q_{n}-2_{2}-1} \frac{\varphi^{\prime \prime}\left(x_{j}\right)}{\varphi^{\prime}\left(x_{j}\right)} \Delta^{(n)} x_{j}
$$

As in the proof of Lemma 6 consider

$$
\begin{aligned}
& p_{s}^{(n-k)}=\frac{1}{\ell\left(C_{s}^{(n-k)}\right)} \sum_{\substack{j: C_{j}^{(n+1)} \subset C_{s}^{(n-k)} \\
0 \leqq j<q_{n-2 i}}} \ell\left(C_{j}^{(n+1)}\right),
\end{aligned}
$$

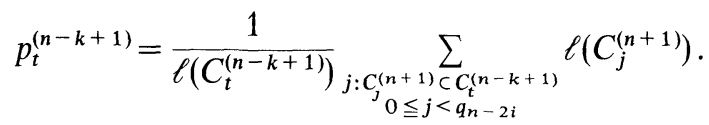

It is easy to see that in the symbolic representation $p_{s}^{(n-k)}, p_{t}^{(n-k+1)}$ has the following form:

$$
p_{s}^{(n-k)}, p_{t}^{(n-k+1)}=\ell(\underbrace{0, A, 0, A, \ldots, 0}_{2 i+1} \mid a_{n-k}, \ldots, a_{0}) .
$$


Then the same arguments as in the proof of Lemma 6 show that

$$
\left|I_{n-2 i}\right| \leqq \text { const } \lambda_{1}^{\sqrt{n}} \text { for } \quad i<\frac{m}{2} \sim \sqrt{\frac{n}{8}} \text {. }
$$

Moreover, for short cycles we have the trivial estimate

$$
\left|I_{n-j}\right| \leqq \text { const } \lambda^{j}, j \geqq m \sim \sqrt{\frac{n}{2}} .
$$

Take now $p \leqq q_{n}$. We can decompose $p$ in the following way,

$$
p=\sum_{i=1}^{\left[\frac{m}{2}\right]} a_{i} q_{n-2 i}+\sum_{j=m}^{n} b_{j} q_{n-j}
$$

where $a_{i} \leqq$ const $n^{2 v+1}, b_{m} \leqq$ const $n^{2 v+1}, b_{j} \leqq \operatorname{const}(n-j)^{v}, j>m$. Using the above estimates we obtain

$$
\left|\sum_{j=0}^{p} \frac{\varphi^{\prime \prime}\left(x_{j}\right)}{\varphi^{\prime}\left(x_{j}\right)} \Delta^{(n)} x_{j}\right| \leqq\left(\sum_{i=1}^{\left[\frac{m}{2}\right]} a_{i}\right) \operatorname{const} \lambda_{1}^{\sqrt{n}}+\text { const } \sum_{j=m}^{n} b_{j} \lambda^{j} .
$$

This estimate gives the desired result. QED.

Remarks. 1. Using a more sophisticated technique one can show that in Lemma 11 the right-hand side can be taken as const $\cdot \lambda_{3}^{k}$. This makes it possible to prove an exponential estimate in Denjoy's lemma and also to consider the rotation numbers $\varrho, \varrho=\left[k_{1}, \ldots, k_{n}, \ldots\right]$, for which $k_{n} \leqq$ const $\cdot B^{n}$ with some $B>1$ as in [8]. It also implies that conjugacy $\psi \in C^{1+\tau}$, for some $\tau>0$.

2. From the viewpoint of the renormalization group ideology the result proved here means the convergence to the linear fixed point of the renormalization group transformation (see [4]) for nondegenerate diffeomorphism of the circle.

\section{References}

1. Vul, E.B., Sinai, Ya.G., Khanin, K.M.: Feigenbaum universality and the thermodynamic formalism. Usp. Mat. Nauk. 39:3, 3-37 (1984) [English transl. Russ. Math. Surv. 39, 1- 40 (1984)]

2. Halsey, T.C., Jensen, M.H., Kadanoff, L.P., Procaccia, I., Shraiman, B.I.: Fractal measures and their singularities: the characterization of strange sets. Phys. Rev. A 33, 2, 1141-1151 (1986)

3. Collet, P., Lebowitz, J., Porzio, A.: Dimension spectrum for some dynamical systems. Rutgers Univ. Preprint

4. Khanin, K.M., Sinai, Ya.G.: Renormalization group method and the K.A.M. theory. In: Nonlinear phenomena in plasma physics and hydrodynamics. Sagdeev, R.Z. (ed.). Moscow: Mir 1986

5. Sinai, Ya.G., Khanin, K.M.: Renormalization group method in the theory of dynamical systems. In: Proceedings of the conference "Renormalization Group 86", Dubna 1986 (in press)

6. Herman, M.: Sur la conjugaison différentiable des difféomorphism du cercle à des rotations. Pub. Mat. I.H.E.S. 49, 5-233 (1979)

7. Herman, M.: Simple proofs of local conjugacy theorems for diffeomorphisms of the circle with almost every rotation number. Bol. Soc. Bras. Mat. 16, 45-83 (1985); 
Sue les difféomorphisms du cercle de nombre de rotation de type constant. In: Papers in Honor of A. Zygmund. Becker, A. et al. (eds.). Belmont: Wadsworth 1983

8. Yoccoz, J.C.: Centralisateurs et conjugaison différentiable des diffeomorphisms du cercle. Thesis Univ. Paris Sud (1985) unpublished;

$C^{1}$ conjugaison des difféomorphisms du cercle. In: Geometry and dynamic. Palis, J. (ed.). Lecture Notes in Mathematics, Vol. 1007. Berlin, Heidelberg, New York: Springer 1983; Conjugaison différentiable des difféomorphisms du cercle dont le nombre de rotation vérifie une condition diophantienne. Ann. Sci. Éc. Norm. Super. 17, 333-359 (1984)

9. Cornfeld, I.P., Sinai, Ya.G., Fomin, S.V.: Ergodic theory. Moscow: Nauka 1980 (English transl:: Berlin, Heidelberg, New York: Springer 1982)

10. Ruelle, D.: Thermodynamic Formalism. Reading: Addison-Wesley 1978

11. Arnol'd, V.I.: Small denominators. I. Mappings of the circumference onto itself. Izv. Mat. Nauk. 25:1, 25-96 (1961) [English Transl. Am. Math. Soc. 49, 213-284]

12. Hawkins, J., Schmidt, K.: On $C^{2}$ diffeomorphisms of the circle which are of type III $_{1}$. Invent. math. 66, 511-518 (1966)

Communicated by E. Lieb

Received January 15, 1987 
